\title{
Application of a new mathematical description of Epidemics to the COVID-19 Omicron wave in few strongly affected countries by the beginning of 2022: USA, UK and France
}

Pavel Petkov ( $\sim$ pavel.p.petkov1956@gmail.com )

\section{Research Article}

Keywords: Linear Differential Equations; Precise solutions; Epidemics peaks; Omicron wave

Posted Date: February 16th, 2022

DOI: https://doi.org/10.21203/rs.3.rs-1363720/v1

License: (a) (1) This work is licensed under a Creative Commons Attribution 4.0 International License. Read Full License 
Application of a new mathematical

${ }^{1}$ STIINTA PENTRU TOTI S.R.L., Dionisie Lupu Str. 33, Bucharest, Romania; ORCID: 0000-0002-3080-9316.

\section{Abstract}

Purpose By means of mathematical modeling, we studied the COVID-19 Omi-

Methods A newly developed [6] mathematical modeling of Epidemics is applied together with a day by day normalization correction accounting for the different number of tests.

Results The new infection cases per day are reasonably described before and around the peak maximum. There are indications that the decreasing part may be well described too. The position of the calculated peaks in time within the Omicron waves indicates everywhere a transition only from the $3^{\text {th }}$ to the $4^{\text {th }}$ fourth the decrease of the new cases per day seem to be related to the combined effect of vaccination and the acquired immunity in a very large part of the population due to Omicron in a short period of time. The calculated cumulative curves support such interpretation.

Conclusions The tracking model describes well the Omicron wave after 


\section{Introduction}

The first signals for the seriousness of the Pandemic COVID-19 on a worldwide scale can be traced back to the beginning of 2020 [1]. Now, in the beginning of 2022, the importance of the issue does not need any argumentation[2]. Much has been learned about the SARS-CoV-2 virus itself, but this process is far to be finished because new virus variants/mutations appear with time, with different properties, including much increased contagiousness in some cases as it is with the Omicron variant identified in late November 2021 [3]. The present work is concerned with some mathematical aspects of modeling the Omicron-stage of the Pandemic which is now dominating or will be soon dominating in most countries of the world [4]. However, one should never forget that dealing with the development of Epidemics is a multidisciplinary subject. Epidemiologists, specialists in infections, virologists, medics with different specializations, pharmaceutics, immunologists, molecular biologists etc. naturally participate by addressing the issues relevant to them. Thus, the modeling remains just a more or less reliable mathematical apparatus for short and/or long term forecasting in order to address more efficiently the issue of the health, social and economic price that societies have to pay to stop/control the Pandemic.

It is important to dispose with mathematical models which, at given fixed conditions, may describe/predict the development of an Epidemics in the full time range till its disappearing (falling in the background). However, in reality this is very difficult simply because conditions do not remain unchanged in a long range scale. Starting from normal season changes, one can arrive to situations where an Epidemics becomes endemic, pathogens undergo evolution or immunity (acquired by recovering from illness or given by vaccination) has a limited duration. To solve such problems, if possible, a class of involved epidemics models, based so far mainly on Ref.[5], are applied, also with many further developments. These models are denoted usually as compartmental models since their idea is to partition the entire population into compartments (groups) with some transition rates between them describing the speed of the displacement of individuals from one compartment to another one. The simplest version is the so-called SIR model where the three compartments are those of the people susceptible to infection (S), the infected people (I) and the number of recovered people (R), all of them being functions of the time $t$. SIR models (as matter of fact the first formulation is in [5]), are supposed to be reliable for infection diseases transmitted from human to human and associated with recovery characterized by lasting resistance to new infections. The dynamics of the model is driven by two transition rates $(S \rightarrow I$ and $I \rightarrow R)$. On the basis of the ideas of SIR, many new model variants were developed. The main directions are attempts to take into account additional population groups with other, different status in the propagation of the infection and the corresponding transition rates. Of special interest are these variants both acquired immunity after recovery and protection via vaccination are of limited duration. At present, it is clear that this is the case of COVID-19 Pandemic for a very large part of the population.

Very recently, we published [6] a new and simple method for describing epidemics including consecutive ways. This method uses also a kind of compartmentalization, where one of the main structures is the group of infected people whose 
different generations are tracked. The other main structure are the generations of recovered people. This compartmentalization naturally appears in the chain of the "human to human to human ..." transmission and represents one of the novelties of the model. In the model, linear differential equations are solved [6] while e.g the SIR-like models require the solving of non-linear differential equations. Only two rates $\lambda_{R}$ (rate of recovery) and $\lambda_{C}$ (rate of infection or spreading rate) are employed which are assumed to be constant in time. A basic prerequisite of such treatment is a large enough population where the spreading of epidemics is governed by statistical laws. In this way, the time for recovery is considered as a random variable when many infected people are considered. In most of the Epidemics-spreading models, the distribution of the recovery times represents an exponential distribution with mean recovery rate $\lambda_{R}=1 / \tau_{R}$ (with $\tau_{R}$ being the mean time for recovery, estimated to about 14 days in the beginning of the Pandemic). In reality, the general population consists of more differentiated groups (we remind the models updating SIR which were discussed previously in the text). The model used [6] does not include these details, it is designed for large enough systems and should work very well at least at the first stage of Epidemics. The goodness of the description depends also of pathogen and immunity related features of the infection. Of course, in relatively small systems, such differentiation is very necessary, as well when networks of clustered infection domains are considered. The model [6] is just a simple tool for a more or less globalized description, whose failure will be seen when indications for the exhausting of the "unlimited reservoir" of susceptible to the infection people appear e.g. when the propagation of the infection suddenly goes downwards. Then, more precise and differentiating methods have to be used after that point of time. The spreading rate of the infection $\lambda_{C}$, is treated similarly to the recovery rate $\lambda_{R}$ i.e. as the mean of a random variable. The important distinction is that $\lambda_{C}$ may be very strongly influenced by measures as confinement, reduction of social contacts, reconfinement, appearance of new virus variants etc. Detailed studies on the epidemics-transmission mechanism and the possibility to control it are given for example in Refs. [7, 8, 9] and references therein. 

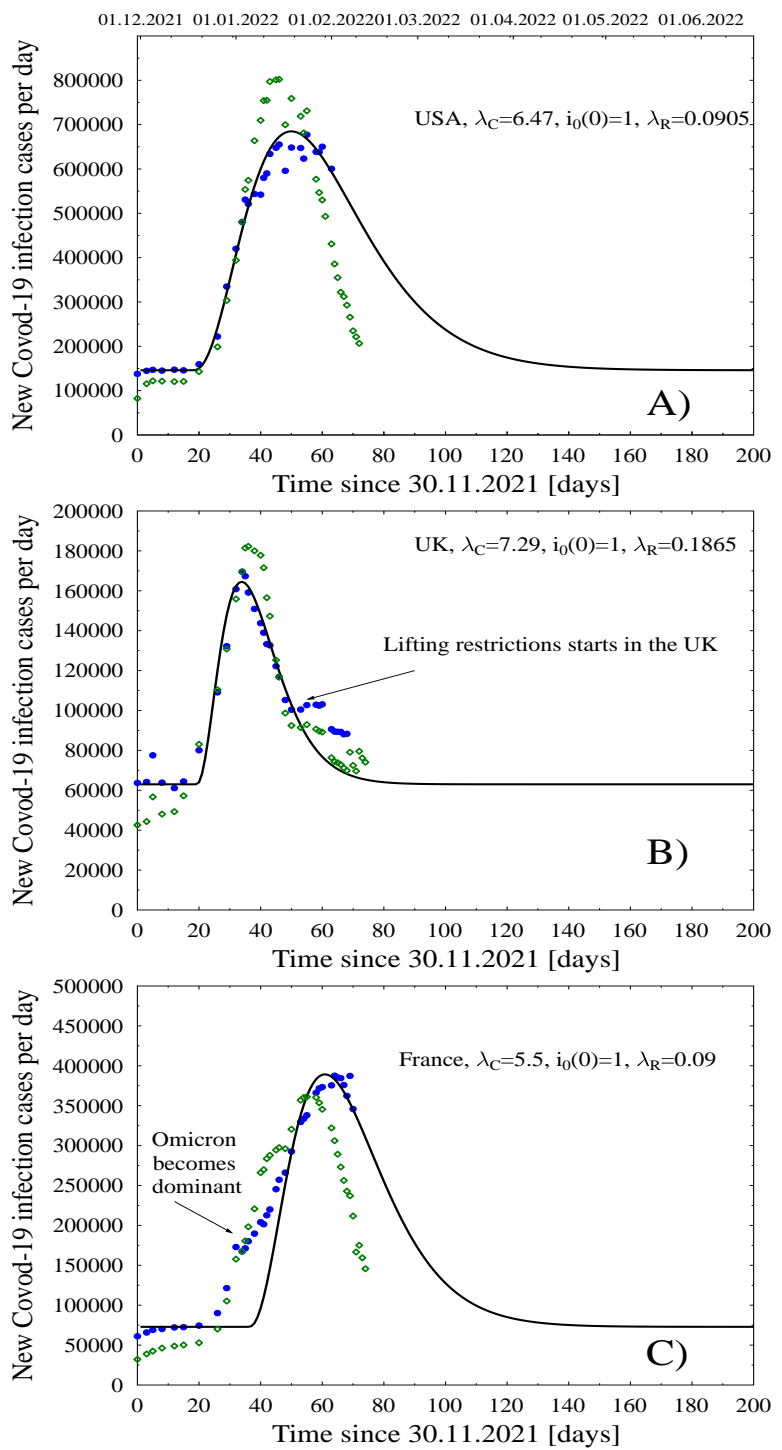

Fig. 1: Part A : Development of the COVID-19 Pandemics in the USA represented by normalized newly registered cases per day (filled circles). The number of tests per day used for a day by day normalization (correction) were taken from the site [10]. The original raw data without normalization are represented by open diamonds. The plateau-like part of the data before the effect of increase is considered as a constant (averaged) background which reflects the previous stage of the Pandemic related developments in the future. The most successful calculation (fit) according to Eq.7 is represented by a continuous line. The derived parameters are also indicated, with the infection and recovery rates in days ${ }^{-1}$ The effect of the normalization is clearly seen and is rather large. An inspection of the data on the number of tests made daily show an non-interrupted reduction trend after reaching the maximum which correlates partly with the very fast decrease of the raw new daily cases. A reduction of the latter cases is predicted also by the calculation but it has a much slower character. Parts B,C: The same as in part A, but for the UK and France, respectively. See also text. 
As already mentioned, the Omicron variant of SARS-CoV-2 is characterized by higher contagiousness than the other dominant previous variants (in the different consecutive time periods), the $\delta$-one "possessing" the previous record. Although in some countries mixing of the $\delta$ and Omicron waves was reported by mid January 2022, in others a very strong effect of increase of the daily new cases indicates the dominance of Omicron. The very simple mathematics in Ref.[6] opens the possibility to forecast the development of Epidemics in time for different infection and recovery rates. This is quite relevant for getting a fast idea for cases related to the appearance of strongly contagious infection pathogens as Omicron. Simultaneously, measures leading to reduce the infection rate may be also taken into account in the modeling of the Epidemics according to [6]. In some disciplines, when one factor dominates all other factors which influence somehow a phenomenon, scientists consider such a case as a testing ground (or even a "laboratory") for checking a particular model and simultaneously learn more about the overwhelmingly dominant factor. Simply the picture at an early stage is quite pure and not obscured by later developments which are likely to occur because of some desynchronizations in the otherwise globalized world. In the present work, we concentrate on three countries where strong increase of the new COVID-19 cases per day was observed since about mid December 2021: the USA, the UK and France.

\section{The method employed for the calculations}

The relevant data sets for the USA, the UK and France were taken from the site [10] and are displayed in Fig.1A,B,C with open diamonds. They cover the time range from 30.11.2021 to 13.01.2022. As discussed in Ref.[6], it is clear that the total population is not tested every day nor the number of people tested each day is the same. Therefore it is necessary to perform a normalization of the raw data for following better in time the absolute number of new daily cases of infections. In this way, fully comparable numbers are considered. However, one has to keep in mind that they are fixed to some constant fraction of the total population (of course, not consistent of one and the same people). There are also factors which may bias the normalization as e.g. possible concentration of tests in regions of the country with higher number of new cases or within clusters of enhanced transmission, reliability of the tests (different brands are used), several tests for one individual in the period considered e.t.c. The number of tests per day used for a day by day normalization (correction) were also taken from the site [10]. The open square are new data points at times larger than the upper limit of the region used for the fits (till 03.01.2022). These points are not normalized because the daily numbers of tests made are very close to each other (of the order of $10^{6}$ and more) in each of the three countries.

The fitting procedure to describe the normalized data is described in details in Ref.[6]. The best results obtained are presented in Fig. 1 with continuous lines in the three panels. The parameters derived are also indicated. Before discussing the results, we remind the basic features of the method. The first step is the determination of the evolution in time of the number of infected individuals $i_{n}(t)$ in the different generations enumerated by $n$. The first generation $i_{1}(t)$ obeys a differential equation 
similar to the well known radioactivity decay law of Rutherford-Soddy:

$$
\frac{d i_{1}(t)}{d t}=-\lambda_{R} i_{1}(t)
$$

with the solution $i_{0}(0) e^{-\lambda_{R} t}$. For the next generations, the linear differential equation for the derivative (change per unit time) of $i_{n}(t)$ contains on the r.h.s. the decrease of $i_{n}(t)$ and the increase due to infections from the previous generation $i_{n-1}$, always with the same rates. The final result for the $n^{\text {th }}$ generation reads:

$$
i_{n}(t)=\frac{\lambda_{C}{ }^{n-1} i_{1}(0) t^{n-1} e^{-\lambda_{R} t}}{(n-1) !}
$$

By summing up the number of infected people from different generations one obtains for their total number as function of time the expression

$$
i(t)=\sum_{k=1,2, . . N_{\max }} \frac{\left(\frac{\lambda_{C}}{\lambda_{R}}\right)^{k-1} n_{1}(0) x^{k-1} e^{-x}}{(k-1) !}
$$

where $N_{\max }$ is the last generation and $x=\lambda_{R} t$. This expression represents a sum of Poisson distributions weighted by the factors $\left(\frac{\lambda_{C}}{\lambda_{R}}\right)^{k-1}$. The quantity defined as $R_{0}=\lambda_{C} / \lambda_{R}$ is the so-called basic reproductive number in the framework of the present model. This reproductive number is somewhat model-dependent and is discussed in detail in $[11,12,13,14]$. Roughly, this is the number of people which will be infected by one contagious individual till the moment when this individual completely recovers. Then, Eq. 2 can be rewritten as

$$
i_{n}(t)=\left(R_{0}\right)^{n-1} \frac{i_{1}(0)\left(\lambda_{R} t\right)^{n-1} e^{-\lambda_{R} t}}{(n-1) !} .
$$

It is to be noted that in the formalism presented the dependence on $R_{0}$ is very strong, changes with the generation number $\mathrm{n}$ and is different from the dependence on $R_{0}$ involved in the other models designed to describe Epidemics in time so far.

Let us continue with the description of some other features. It can be shown that the function $i_{n}(t)$ has one maximum which occurs at

$$
, t_{\max }=(n-1) / \lambda_{R}=(n-1) \tau_{R}
$$

If one considers $i_{n}(t)$ as a distribution over time, its expectation (mean) value M1= $\mathrm{n} \tau_{R}$ is positioned in time after the maximum of $i_{n}(t)$, the difference being just $\tau_{R}$. This means that the epidemics cannot be considered by far as finished even after $t_{\max }+\tau_{R}$. The estimate for the time range of an Epidemics depends on $\tau_{R}$ and $\mathrm{n}$, and increases with $\mathrm{n}$. 
The height of the peak maximum at $t_{\max }$ is given by

$i_{n}\left(t_{\max }\right)=\left(\frac{\lambda_{C}}{\lambda_{R}}\right)^{n-1} i_{1}(0) \frac{(n-1)^{n-2}}{(n-2) !} e^{-(n-1)}=\left(R_{0}\right)^{n-1} i_{1}(0) \frac{(n-1)^{n-2}}{(n-2) !} e^{-(n-1)}$.

285

and its value depends extremely strong on the ratio $\left(\lambda_{C} / \lambda_{R}\right)$ at the power of $(n-1)$. Then, the peak position of $i_{N_{\max }}(t)$ dominates the peak position of the sum of all generations of infected people $i(t) o$.

Concerning the daily behavior of newly infected people (i.e. the new infections per day) used to monitor the epidemics, it can be calculated as

where $\mathrm{K}_{\text {max }}$ sets a limit in the generation number depending on the actual status of the epidemics at time t. The function $C(t)$ can be directly compared to the normalized data on the new cases per day in the Epidemics and thus was used to fit the data in Fig.1. To fix $K_{\max }$ for a particular data set one can use the criteria of correct reproduction of the peak position, shape and volume together with the derivation of the 


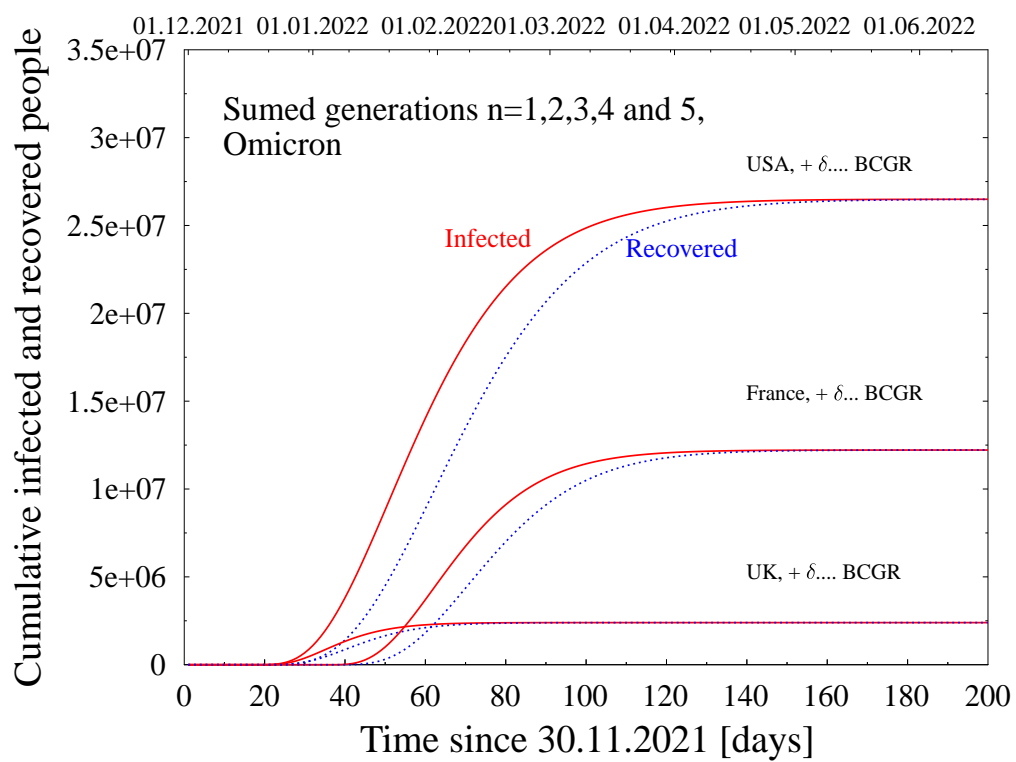

Fig. 2: Cumulative number of infected (continuous curve) and recovered (dotted These curves are closely related to fits of the data on the new infection cases per day shown in Fig.1. The cumulative curves represent the total number of people who have been infected and of the people who recovered. The cumulative curve for the recovered is shifted to larger times compared to the curve for the infected (just by $\tau_{R}$. These curves deal only with the new, more contagious Omicron variant. At large times, the contribution from the background (BCGR) due to $\delta$ and other variants has to be added to the curves to yield the total number of infected and recovered people, and not only the ones related to the Omicron variant (curves displayed on the figure). See also text.

\section{Discussion of the results}

\subsection{Daily new cases of infections}

The first impression coming from Fig.1 is that the quality of the reproduction of the normalized data is very good in all three cases: USA, the UK and France. The role of the normalization is also clearly seen. It was done with respect to the point at 03.01.2022. Without normalizing the open diamonds presenting the raw data in the period before 20.12.2021 would display an artifact-like time behavior. It is amazing that many normalized points fit so well with the fitted curves in the three cases, and especially around the maxima of the fitting curves. Some strong systematic deviation downwards of the raw data from the calculated curves are observed after the peak at much larger times for the USA and France. May be the normalized data will fit better with the calculated curves but this remains to be seen after that the daily tests in the beginning of February become available (they were not at the time when the 
article was written). Some local effects in the data displayed in Fig. 1 can find an explanation in well known real event. Thus, in the UK (panel B) the calculated curve reproduces nicely the normalized points up to the kink up of the data which coincides in time approximately with the massive begin of lifting restrictions scheduled for the week after January $21^{\text {th }}$. In principle, this can be described as switching on of a new wave with modified infection rate $\lambda_{C}$. In the case of France (panel C), again the normalized data points are described reasonably around the maximum (and probably beyond). About the moment were Omicron becomes the dominating variant (about the end of 2021) and wins gradually over $\delta$, the increasing trend of the normalized data approaches the calculated curve. In Table 1, the results of the fits are summarized. The values of the large infection rates $\lambda_{C}$ in the table can be compared with

Table 1: Parameters resulting from the fits of the new infection cases per day for the USA, the UK and France. The first four columns are self explanatory (the country and the two basic parameters $\lambda_{C}, \lambda_{R}$ followed by the mean recovery time $\tau_{R}$ ). The next two columns display the initial day of the calculation according to Eq.7 and the day when the maximal value (the peak-value) of $C(t)$ is reached. The initial date was treated as adjustable parameter in the calculation of the pure Omicron wave. Column 7 displays the time-difference between the peak day and the initial day. By means of the relation $t_{\max }=\Delta_{\max }=(\mathrm{n}-1) \tau_{R}$, the number of the actual dominating generation is derived in the last two columns. It is the same for all three countries. See also text.

\begin{tabular}{|l|l|l|l|l|l|l|l|l|}
\hline Country & $\begin{array}{l}\lambda_{C} \\
{\left[\text { days }^{-1} \text { ] }\right.}\end{array}$ & $\begin{array}{l}\lambda_{R} \\
{\left[\text { days }^{-1} \text { ] }\right.}\end{array}$ & $\begin{array}{l}\tau_{R} \\
{[\text { days }]}\end{array}$ & $\begin{array}{l}\text { Omicron } \\
\text { start Day }\end{array}$ & $\begin{array}{l}\text { Peak } \\
\text { Day }\end{array}$ & $\begin{array}{l}\Delta_{\max } \\
\text { Days }\end{array}$ & $\mathrm{n}-1$ & $\mathrm{n}$ \\
\hline USA & 6.47 & 0.0905 & $\approx 11$ & 18 & 50 & 32 & 3 & 4 \\
\hline & & 0 & & 18.12 .2021 & 19.01 .2022 & & & \\
\hline UK & 7.29 & 0.1865 & $\approx 5.4$ & 19 & 34 & 15 & 3 & 4 \\
\hline & & 0 & & 17.12 .2021 & 03.01 .2022 & & & \\
\hline France & 6.77 & 0.115 & $\approx 8.7$ & 36 & 61 & 25 & 3 & 4 \\
\hline & & 0 & & 05.01 .2022 & 30.01 .2022 & & & \\
\hline
\end{tabular}

the much smaller value of 0.71 days $^{-1}$ derived in Ref.[6] for the first wave in the important common features. The positions in time of the maxima of the peaks (more precisely the differences $\Delta_{\max }$ between peak positions and the $\mathrm{t}=0$ internal time i.e. the Omicron start day) of the calculation indicate that the peaks correspond to the $4^{\text {th }}$ generation of infected people. But one should not forget that this generation provokes infections in the $5^{\text {th }}$ generation and in principle on may expect higher numbers in the peak. There is, however, a development which may suppress the rise of the $5^{\text {th }}$ generation. This is the reduction of people susceptible to the infection due to the combined effects of vaccination and a very large number of new infections within few months leading to an acquired immunity for some time for a very large part of the population. Such combined effects are observed, at the moment of writing this 
paper, in some countries as the UK, the USA, Denmark, France e.t.c. In the framework of the present work, a strong signal for this is given by the decrease featured by the data for the new case per day which is clearly seen in Fig. 1 shortly after reaching the maximum of the wave. However, this decrease is much faster than the calculated one. The calculated curves are in reasonable agreement with data after normalization. To further support the somewhat optimistic conclusion on the development of the Pandemic, estimates in absolute numbers of the corresponding part of the total population which has (will have) some protection by end of the next few weeks (months) have to be made. This is also possible, as shown in Ref.[6], using cumulative data on infections and recoveries. The issue is addressed in the next section.

\subsection{Cumulative curves}

In Ref.[6], formulae for the cumulative curves representing the total number of people who have been infected and of the people who recovered were also deduced (Eq.26 and Eq.29 in that paper). For the case of the data analyzed in the present work, they

Table 2: Comparison of data taken from the site [10] with calculated quantities related to the cumulative number of people who have been infected during the COVID-19 Pandemics in the USA, the UK and France starting from 30.11.2021. The first two columns contain the total number of infected people at the indicated dates. Their difference $\Delta$ is displayed in the third column and illustrates mainly the Omicron contribution (plus that of the other still active virus variants.) The fifth and sixth columns contain the values of the cumulative Omicron curve at day 62 (31.01.2022) and the contribution of the other variants at the same day considered as background, respectively. The last column is the sum $\Sigma$ of the quantities in the fifth and the sixth columns. This sum can be compared to the difference $\Delta$ in column 4 . The agreement is reasonable. See also text.

\begin{tabular}{|l|l|l|l|l|l|l|}
\hline Country & $\begin{array}{l}\text { Total cases } \\
\text { by } 30.11 .2021\end{array}$ & $\begin{array}{l}\text { Total cases } \\
\text { by } 31.01 .22\end{array}$ & $\Delta$ & $\begin{array}{l}\text { Calculated } \\
\text { day } 62\end{array}$ & "Background" & $\Sigma$ \\
\hline USA & 48.6 & 75.0 & 26.4 & 15.0 & 9.1 & 24.1 \\
\hline UK & 10.3 & 17.4 & 7.1 & 2.2 & 3.9 & 6.2 \\
\hline France & 7.7 & 19.2 & 11.5 & 4.3 & 4.5 & 8.8 \\
\hline
\end{tabular}

are displayed in Fig.2. These curves are closely related to fits of the data on the new infection cases per day shown in Fig. 1 from which the parameters were derived. The calculations are relevant to a single wave starting with 1 zero-time patient. Adding the "background" due to the $\delta$ variant and other variants (see Fig. 1 before about 20 5.12.2021 and the previous section 3.1) to the values of the calculated curves at a specific day may yield an estimate of the total number of infected people in the frame of the Omicron wave up to that day (the quantity $\Sigma$ in in Table 2). In Table 2 this is done for the date 31.01.2002 (day 62). The differences $\Delta$ between the registered cumulative infection cases and the calculated $\Sigma$ are in reasonable agreement. At the very earlier stage of the Omicron wave the cumulative curve of the UK increases faster than that of France. Later, the situation is inverted. It should be mentioned that 
by end of the period considered in Table 2 millions of tests were performed per day in the three countries $\left(\mathrm{USA} \approx 1.8 \times 10^{6}, \mathrm{UK} \approx 1.4 \times 10^{6}\right.$, France $\approx 1.110^{6}$ ). Nevertheless, 461 estimates of the full number of infected people need corrections to go from the tested "sample" to the whole population. Using the approach from Ref.[6] (see Table.2 in that work), we did calculate such correction factors which range from about 0.29 (UK), via 0.38 (France), to 1.15 (USA). It is very important that these factors depend on the time period considered. The estimates for the period till end of April 2022 are presented in Table 3. It should be mentioned that the estimates given in Table 3

Table 3: Estimates of the total number of people who have been in contact with the Covid-19 virus or have been fully vaccinated till April 2022. The data in columns 2 and 3 are taken from the site [10]. The contribution of the Omicron wave is given column 4 as calculated using the cumulative curves for the number of people who have been infected during the COVID-19 Pandemics in the USA, the UK and France starting from 30.11.2021. The sum of the data in columns 2,3, and 4 is given in column 5 plus a lower limit in brackets assuming that the cumulative number of infected in column 3 has to be multiplied by $3 \div 4$ as often done. In the last column, the population of the countries is displayed. Correction factors were used to calculate the final contribution of the Omicron wave. See also text.

\begin{tabular}{|l|l|l|l|l|rr|}
\hline Country & $\begin{array}{l}\text { Fully vaccinated } \\
\text { till 30.11.2021 }\end{array}$ & $\begin{array}{l}\text { Infected } \\
\text { till 30.11.2022 }\end{array}$ & $\begin{array}{l}\text { Omicron } \int \text { by } \\
\text { end April 2022 }\end{array}$ & $\begin{array}{l}\text { Total by end } \\
\text { April 2022 }\end{array}$ & $\begin{array}{r}\text { Populatiof } 181 \\
482\end{array}$ \\
\hline USA & 199.6 & 48.6 & 56.2 & $304.4(>402)$ & 320 & 483 \\
\hline UK & 46.4 & 10.25 & 3.5 & $60.2(>80)$ & 67.1 & 484 \\
\hline France & 47.1 & 7.72 & 8.9 & $64.0(>79)$ & 65.1 & 485 \\
\hline
\end{tabular}

are much less reliable then the analysis and derived parameters on the new infection cases per day (Sec. 3.1). For example, what we called "background" in Fig. 1 with the Omicron wave on top may not remain constant, new virus variants may interfere and so on. Nevertheless, these findings are consistent with recent warnings of the WHO that after spring of 2022, for example about half of the population of Europe may be gone through a contact with Omicron. By comparing columns 3 and 4 of Table 3, it is evident that in 4 months the Omicron wave leads to the infection of a larger number of people than the nearly two years previous stage of the Pandemic in the USA and France. The difference of these results to the one for the UK is due formally to the larger value of $\lambda_{R}$ and smaller $R_{0}$ for the latter case, which suppresses (formally) the peak height (cf. Eq. 6) and thus the whole integral.

A new approach [6] for description of Epidemics was applied to the COVID-19 Omicron wave in the USA, the UK and France, a group of strongly affected countries in the beginning of 2022. Because the Omicron effects are very strongly expressed there, the simplest version of that model is employed. The new daily observed infec503 
507

508

509

510

511

512

513

514

515

516

517

518

519

520

521

522

523

524

525

526

527

528

529

530

531

532

533

534

535

536

537

538

539

540

541

542

543

544

545

546

547

548

549

550

551

552

the peak maximum. There are indications that even the decreasing part of those data can be well described as it is the case of the UK. These results were made possible by the use of a day by day normalization taking into account the number of tests made each day. The position of the calculated peaks in time relative to the start of the Omicron waves in the three countries indicates everywhere a transition from the third to the fourth generation of infected people. The fact that a maximum is reached without significant number of infections in the $5^{\text {th }}$ generation seems to be related to the combined effect of vaccination and the infection, in a relatively short period of time (4-5months) of a very large part of the population. This latter effect leads, after the recoveries, to a ( temporary) acquired immunity. Thus, the overwhelming part of the population is characterized by some degree of protection against the virus. In the cases investigated cases, the much stronger decrease of the raw data compared to the calculated (fitted) curves based on normalization seems to be related to some gradual reduction of the number of tests performed each day after the peak maximum. Nevertheless, both sets of data give a very positive signal about the developments in the near future. The calculated cumulative curves, i.e. the total number of infected and recovered people as function of time, support such interpretation. Therefore, a forecast for controlling/stopping the COVID-19 Pandemic soon may be issued with moderate optimism. However, the present work represents exclusively a mathematical modeling of the Pandemic and does not deal with any other aspect of health, social or economic character as well with throwing away any possibility for unexpected developments. Yet we hope that the present results will be of some help.

\section{Acknowledgments:}

The author is indebted to Dr. I. Penev for valuable comments and suggestions.

\section{References}

[1] WHO, Corona virus disease (COVID-19). 2020. https://www.who.int/docs/default- source/coronaviruse/situationreports/20200226-sitrep-37-covid-19.pdf?sfvrsn=2146841e-2. Accessed Date: 20 March, 2020.

[2] WHO, Weekly Epidemiological and Operational updates January 2022, 2022. https://www.who.int/emergencies/diseases/novel-coronavirus-2019/situationreports

[3] WHO, Classification of Omicron (B.1.1.529): SARS-CoV-2 Variant of Concern, 2021). https://www.who.int/news/item/26-11-2021-classification-ofomicron-(b.1.1.529)-sars-cov-2-variant-of-concern

[4] WHO, Enhancing response to Omicron SARS-CoV-2 variant, 2022. https://www.who.int/publications/m/item/enhancing-readiness-for-omicron(b.1.1.529)-technical-brief-and-priority-actions-for-member-states

[5] W.O. Kermack and A.G.McKendrick, Proc. Royal Soc. A 115 (1927) 700

[6] P.Petkov, Stud. Appl. Math, 2021: https://doi.org/10.1111/sapm.12477 
[7] S.L.Chang, N.Harding, C.Zacherson, O.C.CLiff and M. Prokopenko, Nature Communications 11 (2020) 5710; https//doi.org/10/1038/s41467-020-19393-6

[8] R.M.Anderson, H.Heesterbeek, D.Klinkenberg and T.D.Hullingsworth, Lancet 555 395 (2020) 931

[9] J.S.Weitz et al., Nature Medicine 26 (2020) 849

[10] https://ourworldindata.org/coronavirus-testing

[11] P.L. Delamater, E.J.Street, T.F.Leslie, Y.T.Yang and K.H.Jacobsen, CDC EID 25 (2019) 17

[12] O. Diekmann, J.A.P. Heesterbeek and J.A.J. Metz, J. Math. Biol. 28 (1990) 365

[13] E.M.Fine, Epid. Rev. 15 (1993) 265

[14] K.Dietz, Stat. Meth. Med. Res. 2 (1993) 23

-Funding:

The author declares that no funds, grants, or other support were received during

The author has no relevant financial or non-financial interests to disclose.

-Author contributions

The article has one author

-Ethical approval, Consent to participate, Consent to publish

Not relevant 